\title{
Nonsurgical Management of an Extraoral Sinus Tract of Endodontic Origin: A Case Report
}

\author{
Rohit A Tekwani ${ }^{1}$, Zinnie Nanda ${ }^{2}$, Kavita Rudagi ${ }^{3}$, Kranthi K Reddy ${ }^{4}$, Rahul Deore ${ }^{5}$, Shilpa Fotani ${ }^{6}$
}

\begin{abstract}
A longstanding odontogenic infection often leads to the formation of suppuration, which is drained either intraorally or extraorally through a sinus tract. Being a rare condition, the extraoral sinus tract of odontogenic origin is usually misdiagnosed as a non-odontogenic infection by physicians. As it is rightly said that a correct diagnosis is three-fourths the remedy, diagnosing the odontogenic extraoral sinus tract is of prime importance for its effective management. So, this case report demonstrates the diagnosis and the non-surgical endodontic management of an extraoral sinus tract.

Keywords: Endodontic management, Extraoral sinus tract, Odontogenic infection.

Journal of Operative Dentistry and Endodontics (2019): 10.5005/jp-journals-10047-0068
\end{abstract}

\section{INTRODUCTION}

Dental caries, trauma, or failed root canal treatment are often responsible for an infection of the pulpal tissue causing the pulp to become necrotic. A diverse mix of anaerobic bacteria colonize the root canals once the intact pulp chamber is breached and a specialized mixed anaerobic biofilm colonizes the walls of the necrotic root canals. Asymptomatic necrosis of the pulp being a common condition, abscess formation usually occurs only when the bacteria and their toxic products gain an entry into the periapical tissues through the apical foramen and induce inflammation and pus formation. ${ }^{1}$

When pressure within the abscess builds up, it tends to perforate ${ }^{2}$ and forms a drainage duct for the suppuration produced by the abscesses, which is commonly referred to as a sinus tract. ${ }^{3}$ The occurrence of sinus tract may be either intraoral or extraoral. Intraoral sinus tract has a frequent occurrence, while extraoral sinus tract is a rare condition. Extraoral sinus tract of odontogenic origin often lacks intraoral symptoms and thus the patient suspects it to be a cutaneous lesion. ${ }^{4}$ Esthetic concern and discomfort caused due to frequent pus drainage on the face by extraoral sinus tract often makes patient to visit either a physician or a dermatologist and rarely a dentist. ${ }^{5}$ In such cases, a misdiagnosis by them can lead to an ineffective and inappropriate management of a sinus tract. A review of several reported cases reveals that patients who were misdiagnosed have had multiple surgical excisions, radiotherapy, multiple biopsies, and multiple antibiotic regimens, all of which have failed, with a recurrence of the cutaneous sinus tract, because the primary dental etiology was never correctly diagnosed or addressed. ${ }^{6}$ Therefore, a correct diagnosis of an odontogenic extraoral sinus tract is of prime importance for its effective management.

The purpose of this study was to report a case of a nonsurgical endodontic management of an extraoral sinus tract to the chin with a 6-months followup.

\section{Case Description}

A 17-year-old female patient was presented to the Department of Conservative Dentistry and Endodontics at our institute, with the chief complaint of a facial lesion (in the chin region).

\footnotetext{
${ }^{1-5}$ Department of Conservative Dentistry and Endodontics, ACPM Dental College and Hospital, Dhule, Maharashtra, India

${ }^{6}$ Department of Periodontics, ACPM Dental College and Hospital, Dhule, Maharashtra, India
}

Corresponding Author: Rohit A Tekwani, Department of Conservative Dentistry and Endodontics, ACPM Dental College and Hospital, Dhule, Maharashtra, India, Phone: +91 9545597788, e-mail: rtekwani2@ gmail.com

How to cite this article: Tekwani RA, Nanda Z, et al. Nonsurgical Management of an Extraoral Sinus Tract of Endodontic Origin: A Case Report. J Oper Dent Endod 2019;4(1):54-56.

Source of support: Nil

Conflict of interest: None

On extraoral examination, a nodular cutaneous lesion was found in the midline of her chin (Fig. 1). The nodule was soft on palpation and it elicited a purulent discharge from it. Its history revealed that the lesion was present for the past 6 months, which was alternatively appearing and disappearing. Patient ignored this problem for past 6 months and then reported to a local dermatologist for excision of the lesion because of an esthetic concern. After proper examination of the patient, the dermatologist referred her to our institute.

On intraoral examination, there was neither any carious lesion nor tenderness on percussion with mandibular anterior teeth (Fig. 2). On pulp vitality testing, right mandibular central incisor (tooth 41) did not respond to the thermal test and the electric pulp test, while a radiographic examination revealed a definitive periapical radiolucency associated with the same tooth (Fig. 3). On thorough case history recording, the patient disclosed a history of trauma to the chin region two years back.

On the basis of clinical and radiological examinations, chronic suppurative periapical periodontitis with an extraoral sinus was diagnosed in relation to tooth 41 .

A nonsurgical endodontic treatment was initiated on tooth 41. Under local anesthesia, the root canal system was thoroughly debrided using Headstorm file of ISO size 20 (Dentsply Maillefer 


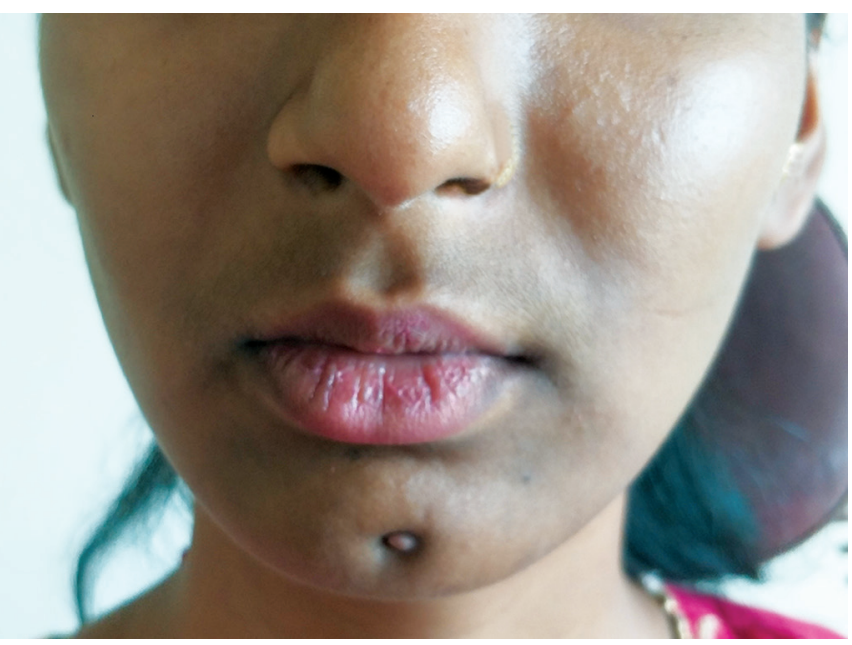

Fig. 1: Preoperative extraoral photograph

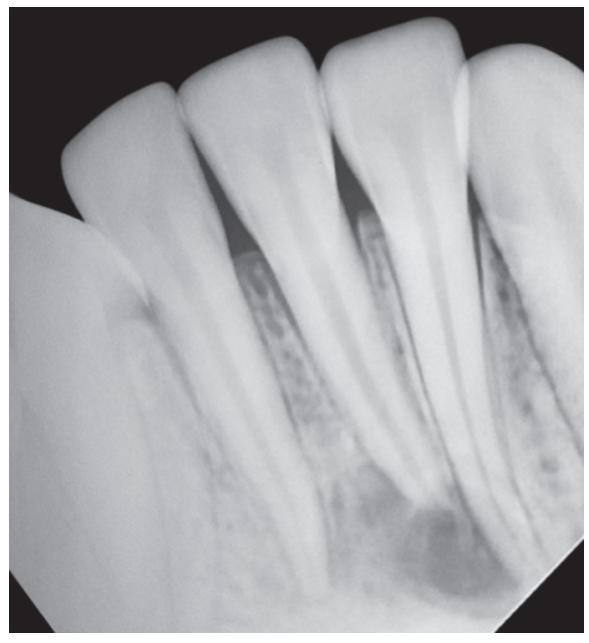

Fig. 3: Preoperative radiograph

Ballaigues, Switzerland). The working length was determined by using Root ZX electronic apex locator (J. Morita, Kyoto, Japan) and confirmed with a periapical radiograph. The root canal system was cleaned and shaped up to K file ISO size 60 (DentsplyMaillefer, Ballaigues, Switzerland) by the step-back technique and irrigated with normal saline. Calcium hydroxide (Ultracal XS; Ultradent, South Jordan, UT) was used as an intracanal medicament for 1 week. After 1 week, there was a sign of healing of the extraoral lesion with no purulent discharge. The canal was then obturated using gutta percha (Dentsply Maillefer, Ballaigues, Switzerland) and AH plus sealer (DentsplyDeTreyGmbH, Germany) (Fig. 4).

\section{FolLOW-UP}

Six months later, the patient was found to be asymptomatic and the extraoral lesion had diminished (Fig. 5), while radiographic examination revealed healing of periapical lesion with respect to tooth 41 (Fig. 6).

\section{Discussion}

A wide variety of disorders such as local skin infections, ingrown hairs, occluded sweat gland ducts, neoplasms, tuberculosis, actinomycosis, and a congenital midline sinus of the upper

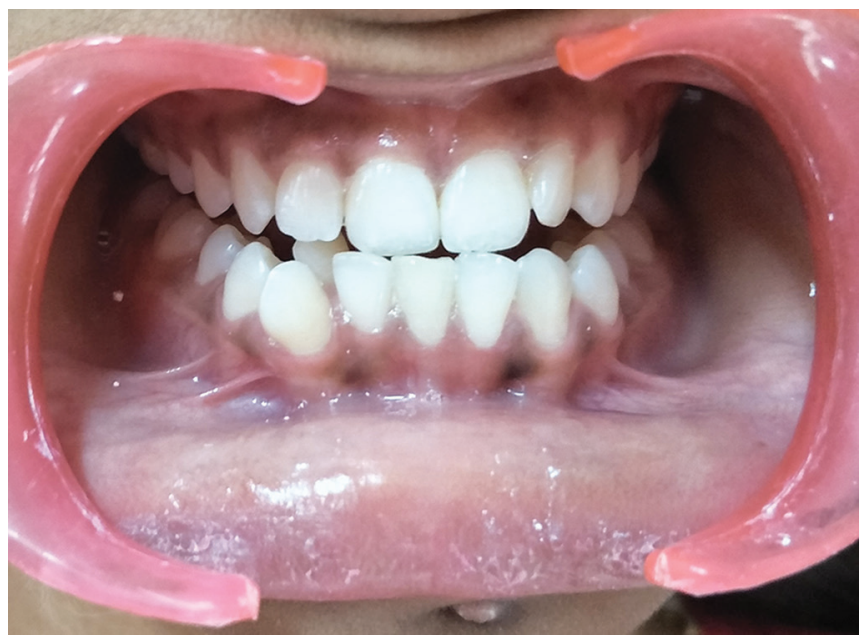

Fig. 2: Preoperative intraoral photograph

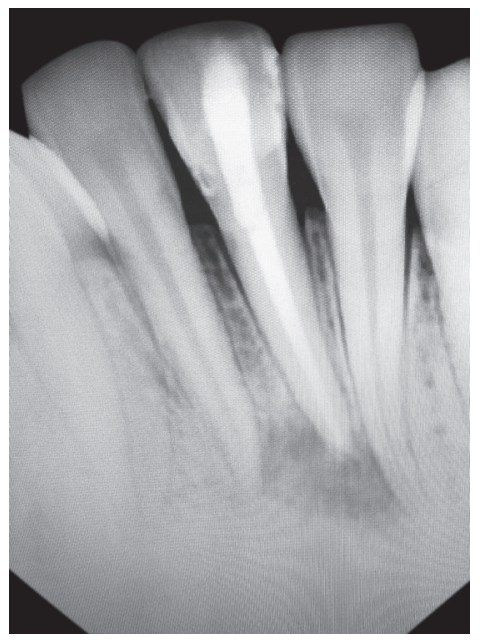

Fig. 4: Radiograph after obturation

lip may be confused with an extraoral sinus tract of dental origin. The possible etiology for development of odontogenic extraoral sinus tract includes trauma to the tooth, retained roots, residual chronic infection of the jaw, and pulpal pathologies.

The purulent exudate of the odontogenic infection will move towards the path of the least resistance from the periapical area. Once the cortical plate has been penetrated, the sinus tract exits as an intraoral or extra oral sinus, depending on the location of the muscle attachments and the facial sheaths. The spread of the infection may be extraoral when the apices of the maxillary teeth are above the maxillary muscle attachments, and for the mandibular teeth below the mandibular muscle attachments. These sinus tracts originally required a surgical intervention apart from the endodontic treatment as they were thought to be lined by epithelium. But later, studies suggested that it was not lined by epithelium but by granulation tissue, which means that it could be intraoral or extraoral, and nonsurgical endodontic treatment could be performed to treat it. ${ }^{4}$

As the sinus tract provides drainage of exudate from the odontogenic primary site, it prevents swelling or pain from pressure build-up. Thus, the draining sinus tract maintains a localized condition and systemic involvement is a rare occurrence. ${ }^{6}$ 


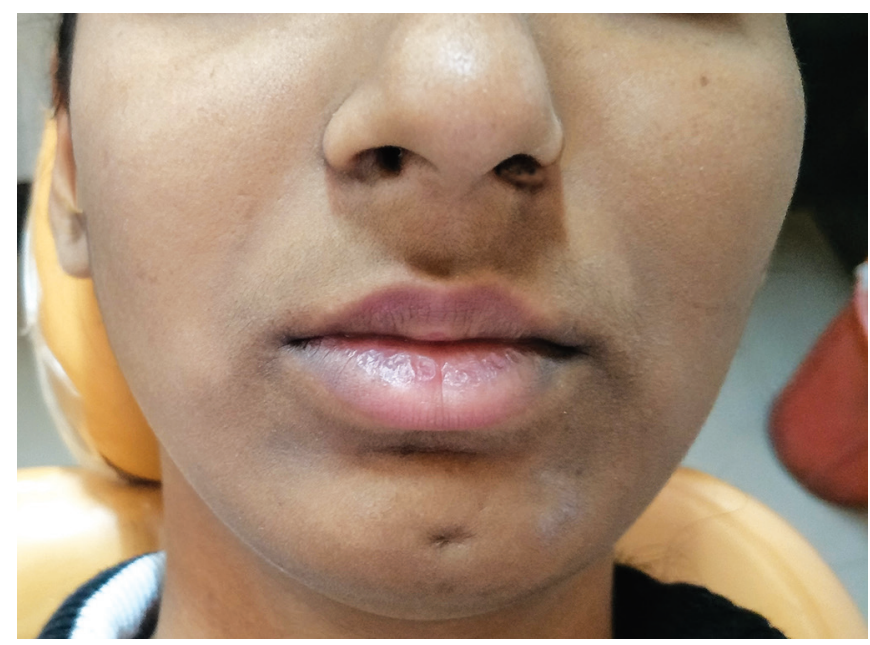

Fig. 5: Postoperative extraoral photograph after 6 months follow-up

The location of the teeth that are involved with the sinus tract can be identified by thorough clinical examination, dental radiography, and sometimes cone beam computed tomography (CBCT), avoiding unnecessary antibiotic and surgical therapies. In order to facilitate diagnosis, a periapical radiography is necessary to demonstrate bone loss in the apex of the infected tooth as on clinical examination a tooth with a necrotic pulp can appear normal or have slightly altered color. Depending on the restorability of the tooth, nonsurgical endodontic therapy (sometimes complemented by a surgery or dental extraction) is the preferred treatment for extraoral sinus tracts of endodontic origin. ${ }^{8}$

As the tooth in this report was restorable, a non-surgical endodontic therapy was initiated. Definitive treatment of the draining sinus tract requires the source of the infection to be completely removed through root canal therapy. A spontaneous closure of the tract should be expected in 5-14 days after root canal therapy or extraction. ${ }^{8}$

In this case, we observed healing of the sinus tract from 4th day of initiation of root canal treatment as calcium hydroxide was given as an intracanal medicament. As there were signs of healing of the sinus tract with the absence of purulent discharge, obturation was done with tooth 41.

In the present case, even after completion of healing, there was a residual dimpling on the chin, but the patient refused to undergo an elective plastic surgery.

\section{Conclusion}

It can be concluded from the presented case that an odontogenic etiology should always be ruled out when patients are presented

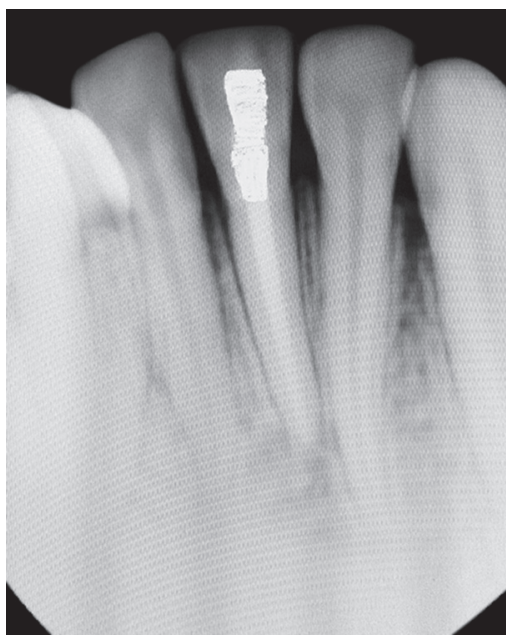

Fig. 6: Postoperative radiograph after 6 months follow-up

with a cutaneous sinus tract on the face, particularly in mandibular, submandibular, and zygomatic regions.

\section{References}

1. Robertson D, Smith AJ. The microbiology of the acute dental abscess. J Med Microbiol 2009 Feb;58(2):155-162. DOI: 10.1099/jmm.0.003517-0.

2. ShamaSA.Periapical abscess of the maxillary teeth and its fistulizations: Multi-detector CT study. Alexandria J Med 2013;49(1):273-279. DOI: 10.1016/j.ajme.2012.10.005.

3. Soares JA, De Carvalho FB, et al. Conservative treatment of patients with periapical lesions associated with extraoral sinus tracts. Aust Endod J 2007 Dec;33(3):131-135. DOI: 10.1111/j.17474477.2007.00058.x.

4. Kumar KS, Subbiya A, et al. Management of an endodontic infection with an extra oral sinus tract in a single visit: a case report. J Clin Diagn Res 2013 Jun;7(6):1247. DOI: 10.7860/JCDR/2013/5369.3064.

5. Johnson BR, Remeikis NA, et al. Diagnosis and treatment of cutaneous facial sinus tracts of dental origin. J Am Dent Assoc 1999 Jun;130(6):832-836. DOI: 10.14219/jada.archive.1999.0307.

6. Tidwell E, Jenkins JD, et al. Cutaneous odontogenic sinus tract to the chin: a case report. Int Endod J 1997 Sep;30(5):352-355. DOI: 10.1111/j.1365-2591.1997.tb00723.x.

7. Choi JU, Lee JK, et al. Submandibular Cutaneous Draining Sinus Tract from Mandibular Osteomyelitis. Korean J Otorhinolaryngol Head Neck Surg 2009 Jun;52(6):549-551. DOI: 10.3342/kjorl-hns.2009.52.6.549.

8. Sotiropoulos GG, Farmakis ET. Diagnosis and conservative treatment of extraoral submental sinus tract of endodontic origin: a case report. J Clin Diagn Res 2014 Oct;8(10):ZD10. DOI: 10.7860/ JCDR/2014/9543.4959.

9. Rudagi KB, Rudagi BM. Cutaneous Sinus Tract of Odontogenic Origin-A Misdiagnosed Lesion: Report of two cases. Acta Stomatol Croat 2012 Dec;46(4):317-322. 